\title{
Operation and Performance of the CMS Silicon Tracker
}

\author{
Ettore Focardi ${ }^{1 \dagger}$ \\ University and INFN Florence, Italy \\ Florence, Italy \\ E-mail: ettore.focardiecern.ch
}

The CMS tracker is the largest silicon detector ever built, covering an area close to $200 \mathrm{~m}^{2}$ and consisting of 15148 silicon strip and 1440 silicon pixel modules. The use of tracker data in physics analysis requires fine-grained monitoring and calibration procedures. Results from timing studies, threshold optimization, calibration of gains and Lorentz angle determination are shown and the impact on resolution and $\mathrm{dE} / \mathrm{dx}$ measurements is discussed. In order to achieve an optimal track-parameter resolution, the position and orientation of its modules need to be determined with a precision of few micrometers and an accurate representation of the distribution of material in the tracker is needed. Results of the alignment of the full tracker are presented, based on the analysis of several million reconstructed tracks recorded during the commissioning of the CMS experiment with cosmic rays and the first proton-proton collisions. They have been validated by several data driven studies and compared with predictions obtained from a detailed detector simulation. Reconstructed photon conversions and nuclear interactions have been used for a first estimate of the tracker material.

Workshop on Discovery Physics at the LHC-KRUGER2010

Kruger National Park, Mpumalanga, South Africa

December 05 - 10, 2010

\footnotetext{
$1 \quad$ Speaker

$\dagger \quad$ On behalf of CMS Collaboration
} 


\section{Introduction}

The CMS apparatus [1] aims to cleanly detect the different signatures of new physics at LHC by identifying and precisely measuring muons, electrons, photons and jets over a large energy range. Experience has shown that a robust tracking and a detailed vertex reconstruction within a strong magnetic field are powerful tools for this purpose. The characterization of events involving gauge bosons, $\mathrm{W}$ and $\mathrm{Z}$ and, in particular, their leptonic decays provide clean experimental signals. To fully exploit these signatures the Central Tracker has to provide a good momentum measurement for energetic leptons. The tracker measurements are combined with track segments reconstructed in the outer muon system to extend the kinematic region of a precise muon momentum measurement. One of the critical issues of the Silicon Tracker is the long-term survival after heavy irradiation. The system has been designed to guarantee stable operating conditions for about 10 years of LHC running. The CMS detector has been designed to record and reconstruct pp collisions at $\sqrt{\mathrm{s}}=14 \mathrm{TeV}$ with an instantaneous luminosity of about $10^{34} \mathrm{~cm}^{-2} \mathrm{~s}^{-1}$. The key element of the CMS detector is the superconducting solenoid, $13 \mathrm{~m}$ long with a diameter of $6 \mathrm{~m}$, operated to produce a magnetic field of $3.8 \mathrm{~T}$ within its volume. Inside the solenoid, from outside to inside, there is a brass-scintillator hadron calorimeter, a crystal electromagnetic calorimeter and the all-silicon tracking detector. Outside the solenoid, embedded in the iron yoke, there are the gaseous detectors to identify the muons and reconstruct their trajectories and momenta together with the inner tracker.

\section{The CMS Tracker}

The CMS tracker is composed of a pixel silicon detector with three barrel layers (BPIX) at radii between $4.4 \mathrm{~cm}$ and $10.2 \mathrm{~cm}$ and two endcap disks (FPIX) at each end, and a silicon strip tracker with 10 barrel detection layers extending to a radius of $1.1 \mathrm{~m}$ and $3+9$ disks on each side of the barrel to extend the overall tracker acceptance up to pseudorapidity $|\eta|<2.5$. The Silicon Strip Tracker (SST) is arranged in four subsystems: the inner barrel (TIB), the inner disks (TID), the outer barrel (TOB) and the outer endcaps (TEC). The total active surface is about 200 $\mathrm{m}^{2}$, the largest silicon tracker ever built. The pixel size is $100 \times 150 \mu \mathrm{m}^{2}$ for a total of 1440 modules with 15840 readout chips and $\sim 66 \mathrm{M}$ channels while the strip tracker is equipped with 24440 sensors ( $300 \mu \mathrm{m}$ thick for the inner layers and $500 \mu \mathrm{m}$ thick for the outer layers) with different pitches, from 80 to $180 \mu \mathrm{m}, 15148$ modules and 75000 readout chips leading to about 9.6 Mchannels. In the strip tracker 4 layers in the barrel and 3 rings in the endcaps are equipped with paired sensors with a $100 \mathrm{mrad}$ relative stereo angle among the strips to provide accurate 3-dimensional hits. This granularity was chosen to balance the need for a low occupancy, which is expected to be a few percent at the nominal LHC luminosity, and the requirement to keep the power density, the needed cooling power and the amount of material at the minimum. The basic performance expected for the tracking detector is a transverse momentum resolution of about 1$2 \%$ for muons of $\mathrm{p}_{\mathrm{t}} \sim 100 \mathrm{GeV}$, an impact parameter resolution of about $10-20 \mu \mathrm{m}$ for tracks with $\mathrm{p}_{\mathrm{t}} 10-20 \mathrm{GeV}$ and the ability to reconstruct tracks in hadronic jets with an efficiency of about 85$90 \%$ and a fake rate not exceeding a few percent. A schematic overview of the Tracker design is shown in Figure 1. 


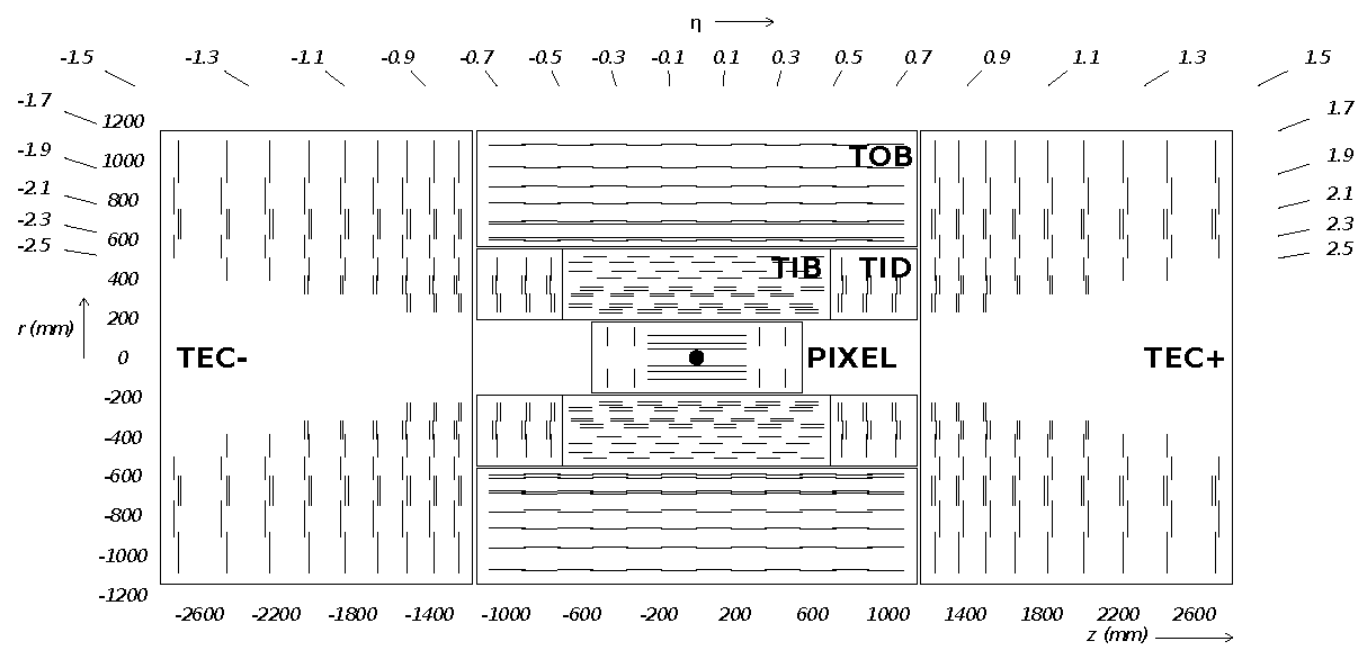

Figure 1: Schematic overview of the CMS tracker. Each line represents a detector module. Double lines indicate double-sided modules which deliver stereo hits.

\section{Detector Operation}

The modules of the pixel and strip detectors have analog read out over optical links, with a digitization off- detector. In the Pixel detector, groups of 4160 pixels (80 rows x 52 columns) are readout by the PSI46V2 readout chip (ROC) [2]. The signal is amplified and shaped with zero suppression performed on the ROC by a comparator for each pixel. When a signal exceeds the comparator tunable threshold it's called a hit and the analog pulse height, the pixel address and the bunch crossing number are stored in buffers for the latency time of the CMS first level trigger. The ROC chip reads out a single bunch crossing. The trigger validates the hits that are then sent to the pixel data acquisition system if the bunch crossing number of the hit and the trigger match. The analog electrical signals from the ROCs are optically converted and sent to the digitizers, Front End Driver (FED) [3].

In the Strip detector, data from groups of 128 strips are collected by APV25 readout chips [4] that perform signal shaping and buffering on the module itself. Each channel of the APV25 has a 192 cell deep pipeline buffer which is used to store the data up to $4 \mu$ s awaiting the level 1 (L1) trigger accept signal. The APV25 has two different readout modes: peak and deconvolution. In peak mode a shaped signal from the detector with rise time of $50 \mathrm{~ns}$ is stored in the pipeline buffers, this mode increases the robustness against timing misalignments and it is suitable for the commissioning and low luminosity data taking. In Deconvolution mode a three sample finite impulse response filter reduces the signal rise time to $12.5 \mathrm{~ns}$ and contains the whole signal in a single bunch crossing; this mode is the default mode for most of the data taking.

After the reception of $\mathrm{L} 1$ accept, the analog data from the corresponding pipeline cell are readout and passed to a multiplexer unit that combines data from 2 APV25 chips and encodes pipeline address, status bits along with the data payload. This data frame is then transferred via a single optical fiber to the digitizers, FED units [5], for further processing. 
The 15148 modules of the Strip Tracker are powered by 983 A4601H CAEN dual channel power supply modules [6]. They are grouped in 29 crates and controlled by 8 SY2527 CAEN mainframes. The total power delivered to the tracker varies between 30-60 kW depending on the operating conditions. The PS modules provide 1.25 and $2.5 \mathrm{~V}$ and a bias voltage (HV) up $600 \mathrm{~V}$ to the modules. The transition HV off-on can be done in $\sim 75 \mathrm{~s}$. The HV of the system is turned $\mathrm{ON}$ after the LHC has declared the beam conditions to be stable.

The 1440 Pixel modules are powered by 50 A4603 CAEN dual channel modules, grouped in 10 crates and controlled with 1 SY1527 CAEN mainframe.

An efficient cooling of the Tracker modules is essential due to the amount of power generated by the Tracker and to mitigate the effect of the radiation damage on the sensors and electronics caused by large particle fluences at the LHC. The cooling plant is composed of 3 cooling loops in cascade (Chiller group (filled with R507A fluid), Brine(filled with Fluorocarbon $\left(\mathrm{C}_{6} \mathrm{~F}_{14}\right)$ fluid) and 7 detectors cooling plants). The 7 detector cooling units are 2 for Silicon Strips, 1 for Pixel, 2 for Thermal screen, 2 for Preshower (Electromagnetic calorimeter). The cooling fluid is $\mathrm{C}_{6} \mathrm{~F}_{14}$ and the plant is designed to operate between room temperature and $-20{ }^{\circ} \mathrm{C}$. The current operating temperature is $+4{ }^{\circ} \mathrm{C}$. To keep the humidity in the Tracker volume always well below the dew point for any given operational temperature, the detector volume is flushed with nitrogen. During the last two years operation few incidents occurred that caused some leaks in one of the strips cooling plant (SS2), 4/180 lines are closed but the modules are operational. Inside the Tracker volume we measured a content of $\sim 0.6 \%$ in volume of $\mathrm{C}_{6} \mathrm{~F}_{14}$.

\section{Detector Commissioning}

The commissioning of the detector requires a series of operations. In the Pixel detector we calibrate the readout chain: timing, output gain, laser settings for optical readout links; calibrate the response pixel by pixel, optimize the zero suppression threshold, do fine delay timing scan with collisions to maximize efficiency and cluster size (Fig. 2).
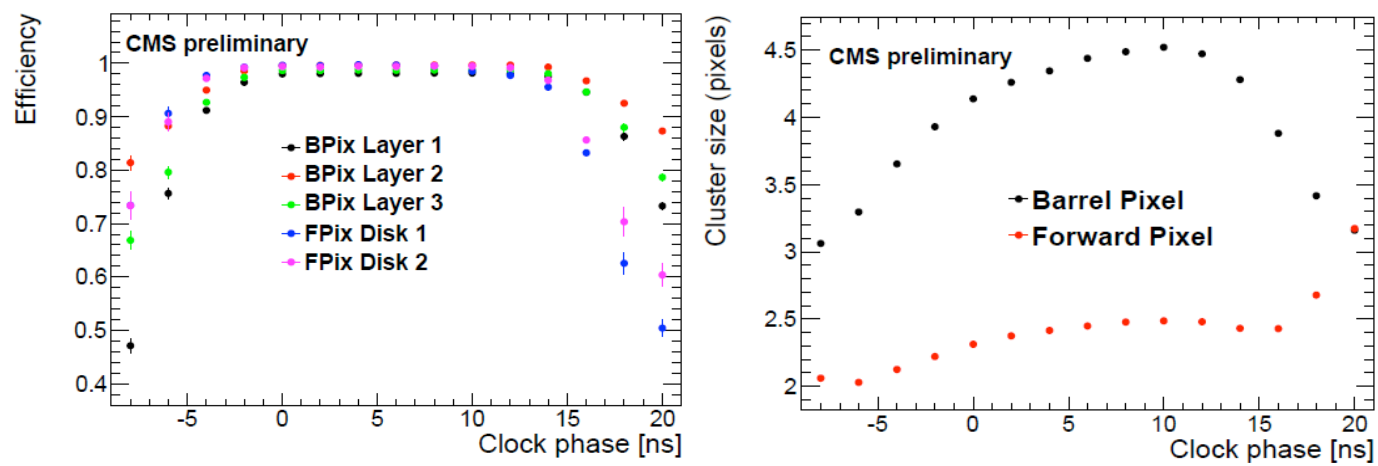

Fig.2 Fine delay timing scan for pixel to maximize efficiency and cluster size performed with $7 \mathrm{TeV}$ collisions. 
In the strip detector the lasers tuning for optical readout links is done, followed by a readout chip optimization for pulse shape and analog baseline. Then noise and pedestal measurements strip by strip are performed followed by a synchronization on module level. At the end a fine timing delay scan is done.

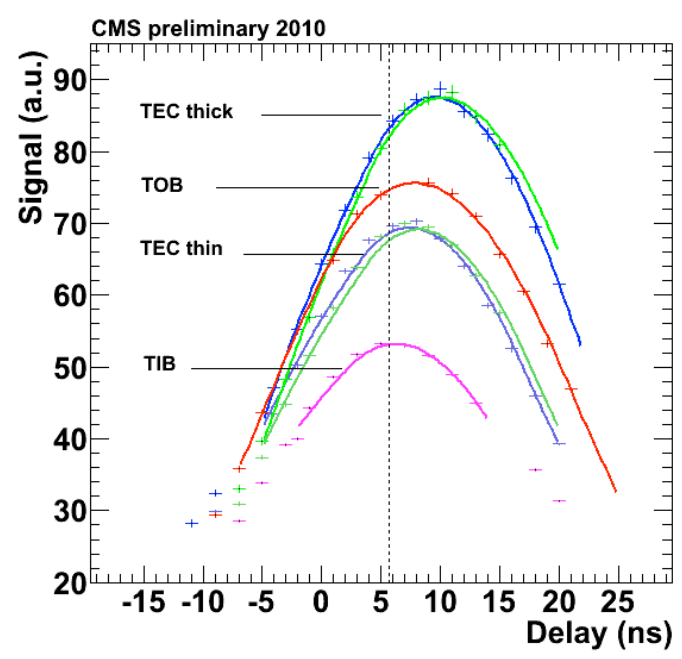

Fig.3 Timing scan for strips performed with $7 \mathrm{TeV}$ collisions

Scanning the signal peak with collision data allows to correct the synchronization to $1 \mathrm{~ns}$ level, Fig.3. These operations aim to maximize the signal identification and tracking efficiency for intime particles and minimize pileup due to out-of-time particles.

\section{Detector Performance}

After the full commissioning chain, the operational fraction of the silicon tracker is $98.3 \%$ for the Pixel detector and $97.2 \%$ for the Strips Tracker. The data taking with CMS started in 2008 and $\sim 8 \mathrm{M}$ tracks from cosmic muons were collected by the Tracker in 2008-2010. In 2009 300 $\mathrm{K}$ minimum bias events were collected with pp collisions at $\sqrt{\mathrm{s}}_{\mathrm{s}}=900 \mathrm{GeV}$ and $2.36 \mathrm{TeV}$. The number of events in pp collisions collected in 2010 at $\mathrm{E}=7 \mathrm{TeV}$ was corresponding to $\sim 43 \mathrm{pb}^{-1}$. The reconstructed hit signals match with the expectation. The strips and pixel clusters are formed from adjacent strips and pixels with charge over the readout threshold. The strip clusters signal-to-noise ratio $(\mathrm{S} / \mathrm{N})$ is evaluated from on-track clusters and corrected for the incident angle. The results are a $\mathrm{S} / \mathrm{N} \sim 19$ for thin sensors and $\mathrm{S} / \mathrm{N} \sim 23$ for thick sensors. The efficiency in the strip tracker is measured as follows: the pattern recognition is repeated excluding all hits from the module under study. For tracks passing through this module, it is checked if a hit consistent with the expected position from the extrapolation of the track direction is found. All modules are used, then the procedure is repeated excluding faulty modules. The efficiency for the pixel detector is measured by the ratio hits found/hits expected. The Pixel and strip hit efficiency is shown in Fig.4.

The intrinsic position resolution is measured using tracks that cross the sensor overlap region in the barrel layers. For the strips the resolution spans an interval between 17.2 and $39.6 \mu \mathrm{m}$ for 
respectively inner and outer layers. For the pixel in the transverse $r \phi$ coordinate results $12.7 \pm 2.3$ $\mu \mathrm{m}$ and in the longitudinal $\mathrm{z}$ coordinate is $28.2 \pm 1.9 \mu \mathrm{m}$. The alignment of the pixel and strip detector is performed using two algorithms in sequence: a global method, Millipede II [7], which extrapolates the real module position from a global residual minimization, and a local method, Hit and Impact point (HIP) [8], which performs a residual minimization assuming no track parameter dependance.
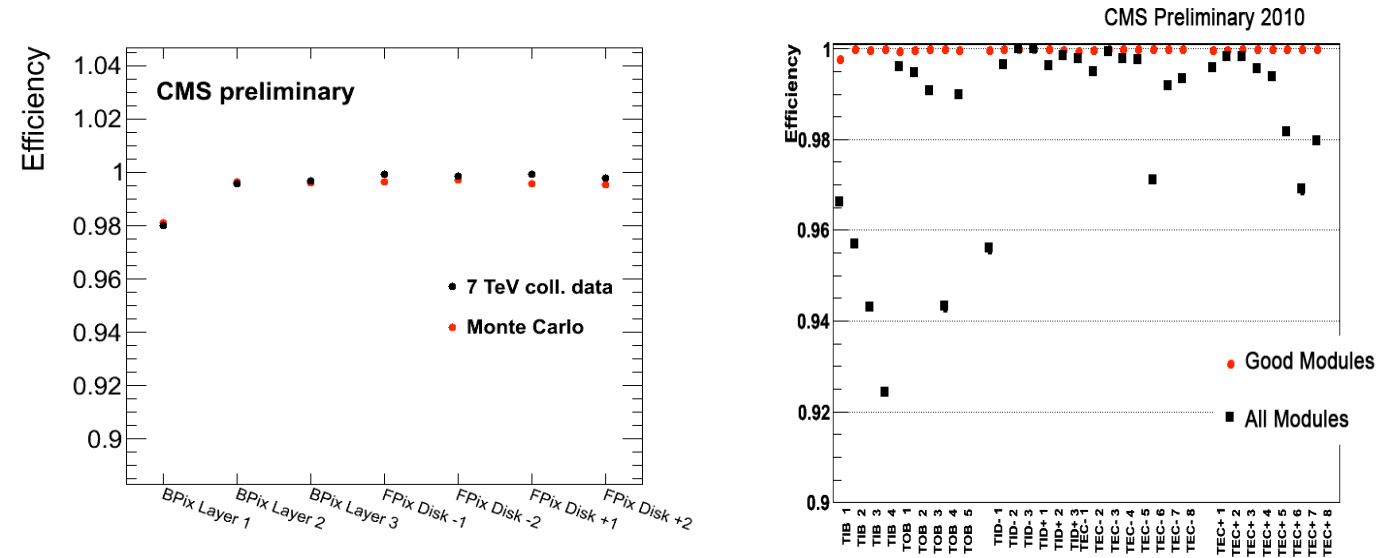

Fig.4 Pixel (left) and strips (right) efficiency

The correlations between modules are solved through multiple iterations. The RMS of the distribution of the median of the residuals estimated with tracks from minimum bias events ( $p>3$ $\mathrm{GeV} / \mathrm{c}$ ) was found to be $1.6 \mu \mathrm{m}$ in the pixel barrel and $5 \mu \mathrm{m}$ in the inner strip barrel, Fig. 5 .
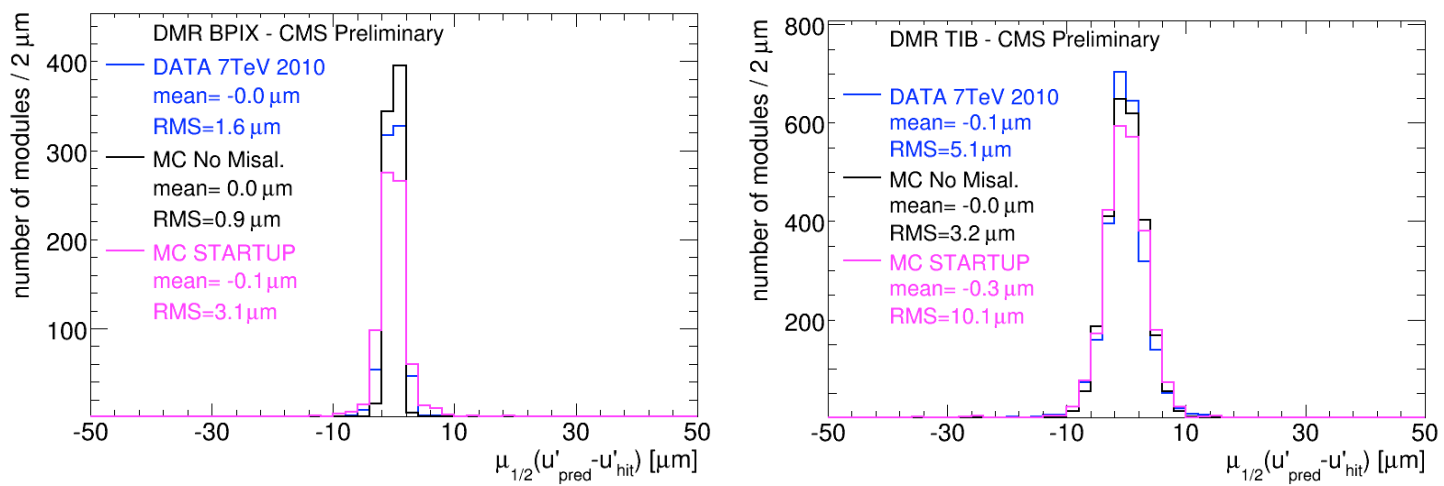

Fig.5 Distributions of the hit residual medians for sensors with more than 200 hits in barrel pixel (left) and inner strip barrel (right).

To ensure good performance of the Tracker detector and high data quality a data quality monitoring (DQM) is used, online and offline. The online DQM samples $10 \%$ of the events due to bandwidth limitations while the offline DQM uses the entire data set. Results of processed data are circulated in summary reports that can alert in case of deviation of distributions from the expected behavior. 


\section{Conclusions}

The operation and performance of the CMS Tracker have been described. The detector has been recording efficiently high quality data during the first year of pp collision runs with very good operational status of $\sim 98 \%$. The Pixel cluster charge and the high strips $\mathrm{S} / \mathrm{N}$ ratio are in agreement with the expectations. The single hit efficiency is close to $100 \%$ and the alignment is already at level of a few microns. A multi stage data quality monitoring is in place, providing quick feedback to the operations.

\section{References}

[1] CMS Collaboration, “The CMS Experiment at CERN LHC”, JINST 3, S08004 (2008)

[2] H.C. Kästli et al., Nucl. Instr. and Meth. A565(2006)188

[3] M. Pernicka et al., Topical workshop on Electronics for Particle Physics, Prague, Czech Rep., $03-$ 07 Sept 2007, 487, CERN-2007-007

[4] L. Jones et al.,'The APV25 deep submicron readout chip for CMS detectors', $5^{\text {th }}$ workshop on electronics for LHC experiments (LEB99), Snowmass, Colorado, 20-24 Sept. 1999, CERN-1999-09

[5] C. Foudas et al., IEEE Trans. Nucl. Science 52(2005)2836

[6] S. Paoletti et al., 'The powering scheme of the CMS Silicon Strip tracker', $10^{\text {th }}$ Workshop on electronics for LHC and future experiments, CERN-2004-010.

[7] V.Blobel, Software alignment for tracking detectors,Nucl.Instrum.Meth.A566(2006)5; A program description for Millepede II and the code is available via: http://www.desy.de/ blobel.

[8] V. Karimäki, T. Lampén and F.-P. Schilling, The HIP Algorithm for Track Based Alignment and its Application to the CMS Pixel Detector, CMS-NOTE-2006-018. 